Communications in Physics, Vol. 28, No. 2 (2018), pp. 189-190

DOI:10.15625/0868-3166/28/2/10078

\title{
Comment paper
}

\section{REBUTTAL TO M.E. HASSANIS FOUNDATIONS OF SUPERLUMINAL RELATIVISTIC MECHANICS}

\author{
A. $\operatorname{SFARTI}^{\dagger}$ \\ University of California, Berkeley (Soda Hall), Berkeley, California, USA \\ ${ }^{\dagger} E$-mail: egas@pacbell.net
}

Received 13 June 2017

Accepted for publication 31 March 2018

Published 16 June 2018

\begin{abstract}
M.I. Hassani tries to construct the foundations for a superluminal mechanics. In the following note we will prove the premise of his paper to be wrong, rendering his whole paper as incorrect.
\end{abstract}

Keywords: superluminal relativistic mechanics.

Classification numbers: 03.65.Ud.

\section{INTRODUCTION}

The starting point of M. Hassanis paper is the statement that [1]:

In order to avoid this singularity, one can simply prohibit the existence of luminal inertial reference frames (IRFs),that is to say, a set of inertial frames that may be in rectilinear uniform motion at luminal velocity relative to each other. But such a prohibition seems to be entirely unreasonable because in the Nature; none can prevent any free material body from reaching or exceeding light speed in vacuum.

The last sentence is the basis of the Hassani paper and it can be proven false by a simple application of dynamics. 


\section{DISPROOF}

The mechanical work $W$ exerted on a particle of mass $m$ over a distance $L$ by a force $F$ is:

$$
W=\int_{r=0}^{r=L} F d r
$$

We know that:

$$
F=\frac{d p}{d t}=m \frac{d(v \gamma(v))}{d t}
$$

Thus

$$
\begin{aligned}
W & =\int_{r=0}^{r=L} m \frac{d(v \gamma(v))}{d t} d r \\
& =\int_{v=0}^{v=V} d(v \gamma(v)) \frac{d r}{d t} \\
& =m \int_{v=0}^{v=V} v d(v \gamma(v))
\end{aligned}
$$

When $r$ varies from 0 to $L$ the speed $v$ varies from 0 to $V$. We know that:

$$
d(v \gamma(v))=\gamma^{3}(v) d v
$$

So

$$
W=m \int_{v=0}^{v=V} v \gamma^{3}(v) d v=m c^{2}(\gamma(V)-\gamma(0)) .
$$

For $V=c, \gamma(V)=\infty$ and $W=\infty$ so the starting point of Hassanis paper is wrong.

\section{CONCLUSION}

The premise of the Hassani paper [1] has been shown to be wrong, rendering the whole paper incorrect.

\section{REFERENCES}

[1] M. E. Hassani, FOUNDATIONS OF SUPERLUMINAL RELATIVISTIC MECHANICS, Communications in Physics, 24 (4) (2014) 313-332. 